\title{
Estratégias para Alocação de Recursos de Controle Ótimo em Cenários Estocásticos
}

\author{
Arlindo R. Galvão Filho ${ }^{1}$, Telma W. Lima Soares ${ }^{1}$, Clarimar José Coelho ${ }^{2}$ \\ ${ }^{1}$ Instituto de Informática - Universidade Federal de Goiás (UFG) \\ Câmpus Samambaia - 74.690-900 - Goiânia - GO - Brazil \\ ${ }^{2}$ Escola de Ciências Exatas e da Computação \\ Pontifícia universidade Católica de Goiás (PUC-Goiás) - Goiânia - GO - Brazil \\ \{argfilho,clarimarc\}@gmail.com, telma_woerle@ufg.br
}

\begin{abstract}
Application of computational models to epidemiology has contributed to the dynamic understanding of various infectious diseases. Simulations and predictions using such models are being used in numerous public health policies against the current COVID-19 pandemic. Three widely used examples are deterministic formulations of compartmental models, based on individuals and based on complex networks. In this context, this work proposes studies on a stochastic perspective of these models in order to insert uncertainty into the epidemiological dynamics, as well as to obtain optimal control strategies for their mitigation. The results show significant reductions in the number of infected individuals.
\end{abstract}

Resumo. A aplicação de modelos computacionais à epidemiologia tem contribuído para o entendimento da dinâmica de várias doenças infecciosas. Tais modelos estão sendo usados para simulações e predições em várias políticas de saúde pública contra a atual pandemia do COVID-19. Três exemplos amplamente utilizados são formulações determinísticas dos modelos compartimentais, baseados em indivíduos e baseados em redes complexas. Neste contexto, este trabalho propõe estudos sobre uma perspectiva estocástica destes modelos no intuito de inserir incerteza à dinâmica epidemiológica, bem como a obtenção de estratégias de controle ótimo para sua mitigação. Os resultados mostram reduções significativas na quantidade de indivíduos infectados.

\section{Introdução}

A teoria do controle ótimo é usado para encontrar uma lei de controle para um sistema dinâmico durante um período de tempo de modo a otimizar uma função objetivo onde o sistema dinâmico seja, por exemplo, uma epidemia [Luenberger 2010, Molter et al. 2016]. Uma epidemia pode se propagar rapidamente pela população e persistir durante longos períodos de tempo na comunidade. Em casos extremos, a proliferação de uma epidemia pode afetar a civilização mundial como ocorre no momento com a COVID-19 [Yi-Chia et al. 2020]. A atual dinâmica social (globalizada) impõe a análise das doenças e das redes que as transmitem. Os padrões de propagação das epidemias por pessoas são determinadas por propriedades patogênicas da doença e pela estrutura da rede de pessoas afetadas. A propagação ocorre pelo contato entre as pessoas (rede de contato) [Siettos and Russo 2013]. A compreensão da dinâmica da rede 
é crucial para conhecer a propagação da epidemia. As redes de contato, mesmo dentro do grupo populacional podem ser divergentes quando as doenças também são divergentes. Tal divergência depende das diferentes vias de transmissão do agente patológico [Choisy et al. 2007]. No caso de uma doença muito contagiosa, onde a transmissão pode ocorrer pelo ar por inalação ou contato com gotículas infectadas, a rede tem um grande número de ligações. No caso de uma doença sexualmente transmissível, a rede não é complexa e tem menos ligações [Emilia and White 2010].

Uma das áreas da ciência que tem por objetivo o estudo desses fenômenos associados com a dinâmica das doenças e á saúde das populações é a Epidemiologia. Os fenômenos são avaliados de forma quantitativa e qualitativa. Além disso, a epidemiologia estuda os possíveis fatores de risco que possam condicionar doenças [Filho and Rouquayrol 2006]. Dentro dessa grande área, um importante campo de investigação consiste na chamada epidemiologia matemática, que se caracteriza pelo uso de modelos matemáticos para o desenvolvimento de estratégias de controle e prevenção de epidemias [Anderson and May 1992].

No contexto da epidemiologia matemática clássica são usados modelos compartimentais determinísticos para o estudo de doenças para grande densidade populacional. A modelagem define diferentes estados para representar o indivíduo em relação à doença e acompanhar sua evolução ao longo do tempo. Os estados do indivíduo são definidos como Suscetível (S), indivíduo suscetível a infeção. Infectado (I), indivíduo infectado pela doença e pode transmiti-la a um ou mais indivíduos. Recuperado (R), depois de recuperar de uma infeção o indivíduo passa para o estado recuperado e está imune a possíveis infeções. Os modelos estocásticos estimam as variações de probabilidade dos resultados e permitem variar aleatoriamente uma ou mais entradas ao longo do tempo. Dependem das variações aleatórias do risco de exposição da doença e de outras dinâmicas das doenças. Em geral, modelos aleatórios são usados em flutuações importantes como em populações pequenas [Galvão Filho et al. 2016, Galvão Filho et al. 2017].

A proposta do trabalho é apresentar o potencial das modelagens determinísticas e estocásticas para diversos cenários epidemiológicos. Os estudos levam em consideração diferentes métodos de controle, otimização de recursos e inserção de incertezas nos modelos propostos. Os estudos analisam os modelos compartimentais SIR e SIRC, bem como seu equivalente estocástico no modelo baseado em indivíduos e modelagens em redes complexas. As demais seções do trabalho descrevem, sucintamente, as abordagens propostas e seus resultados.

\section{Resultados e contribuições}

O modelo epidemiológico mais comum divide a população em compartimentos ou classes: Suscetíveis, Infectados e Recuperados (SIR). A dinâmica da epidemia é representada por um conjunto de equações diferenciais determinísticas que descrevem a evolução temporal do número de indivíduos em cada uma das classes/compartimentos [Kermack and Mckendrick 1927]. Este trabalho propôs um modelo SIR para alocação temporal de um controle ótimo para a dinâmica da influenza sazonal utilizando métodos de barreira e com recursos limitados. Verificou-se que o controle ótimo escolhido alocou os recursos na região do horizonte de tempo em que o contágio era mais intenso, quando aplicado instantaneamente. Quando inserido um atraso para a campanha se tor- 
nar efetiva, observou-se que as menores quantidades de indivíduos infectados no decorrer da simulação foram apresentadas adiantando a ação de controle. Os resultados foram publicados em [Galvão Filho et al. 2013].

Outra forma de modelagem epidemiológica, são os Modelos Baseados em Indivíduos (MBI) [Cisternas et al. 2004]. MBIs são modelos estocásticos que representam cada indivíduo da população como uma entidade única e discreta, possuindo ao menos uma característica que muda ao longo do seu ciclo de vida. Permite a inclusão de novas características como nos modelos compartimentais [Lambert et al. 2020]. Além das classes utilizadas no modelo SIR é possível que cada indivíduo tenha uma ou mais características possibilitando a inserção de heterogeneidades individuais ao modelo.

Outro modelo compartimental, é o SIRC, que foi proposto inicialmente para representar a dinâmica da Influenza A [Casagrandi et al. 2006]. Trata-se de um modelo similar ao SIR, porém com um novo estágio intermediário entre um indivíduo susceptível e um recuperado, chamado de cross-imune [Di Giamberardino and Iacoviello 2019]. No SIRC é possível que os indivíduos naturalmente percam a imunidade, sejam expostos mais de uma vez à doença entre outras características que o tornam mais próximo de uma aplicação real.

Em [Galvão Filho et al. 2016] foi demonstrado a dinâmica do modelo SIR bem como uma dinâmica equivalente a ela proveniente de um modelo MBI. Neste contexto, este trabalho propôs a implementação do modelo SIRC baseado em indivíduos equivalente ao modelo compartimental. Os resultados mostram que a equivalência depende diretamente do tamanho da população e do passo de discretização. Cada estado foi analisado individualmente, mostrando que os desvios padrões acompanham os limiares médios. Esses resultados foram publicados por Galvão Filho e colaboradores em [Galvão Filho et al. 2017].

Modelos compartimentais, como o SIR e o SIRC clássicos utilizam abordagens determinísticas, e dessa forma, a análise da política de controle ótimo em questão será avaliada sob as mesmas condições em todas realizações. Assim, seria interessante gerar uma avaliação do controle ótimo, obtido pela modelagem determinística, em um cenário estocástico. Tal procedimento agregaria variabilidade nos resultados, que seriam agora analisados em média, possibilitando a verificação de melhores/piores casos. Acredita-se que é possível agregar uma certa robustez ao modelo.

Neste contexto, foi proposto o uso dos modelos SIR e SIRC determinísticos para obtenção do controle ótimo, e posteriormente avaliação desta política de controle aplicada em um cenário homogêneo utilizando um modelo MBI equivalente. As estratégias de controle escolhidas foram vacinação e método de barreira para o modelo SIR e vacinação e tratamento para o SIRC. Foi aplicado um estudo de caso baseado em uma dinâmica da influenza, em que ambas as estratégias mostraram uma redução na quantidade de indivíduos infectados. Resultados equivalentes foram obtidos nos modelos MBI-SIR e MBI-SIRC com e sem o uso de controle. Os resultados foram avaliados em um estudo de caso baseados na influenza comum e influenza A em relação ao custo gasto pelo controle e seu impacto gerado na população. O modelo MBI-SIR evidenciou uma diferença expressiva quanto ao uso dos controle individualmente em relação do uso em conjunto. Porém, devido ao fato do modelo MBI-SIRC não ter se mostrado tão sensível aos controles uti- 
lizados neste estudo de caso, não foi possível obter uma boa visualização da estratégia a ser utilizada. De acordo com os desvios padrões obtidos não há uma diferença expressiva entre os pesos variados. A análise utilizando o custo gerado pelo controle em conjunto com o impacto dele na população foi de grande valia, uma vez que as simulações não foram feitas com apenas um peso para cada critério de avaliação. Desta forma foi possível verificar 16 cenários para o SIRC e 19 para o SIR. Os artigos contendo resultados obtidos com a avaliação da alocação de recursos de controle nos modelos MBI-SIR e MBI-SIRC estão em fase de submissão (MBI-SIR) e revisão (MBI-SIRC).

Em comparação com modelos compartimentais tais como o modelo SIR e SIRC, o custo computacional para a simulação de um MBI é tipicamente mais elevado. No entanto, este custo é associado principalmente à repetições de operações simples e similares de modo não sequencial. Este cenário é bastante favorável para o uso de plataformas de processamento paralelo, tais como as unidades de processamento gráfico (Graphics Processing Unit, GPU). Neste contexto, este trabalho propôs uma maneira de acelerar as simulações de modelos epidemiológicos baseados em indivíduos utilizando GPUs [Galvăo Filho et al. 2011]. Os resultados obtidos com a paralelização do algoritmo, resultou na redução do tempo computacional por um fator de vinte. Tais resultados foram publicados em [Galvão Filho et al. 2016].

Modelos baseados em redes complexas [Boccaletti et al. 2006] são fundamentados em conceitos matemáticos da teoria dos grafos. Os modelos em rede complexas representam cada indivíduo como um nó e a relação entre um ou mais indivíduos como arestas. Além das diversas ferramentas que a teoria dos grafos proporciona para obtenção de informações na rede, os modelos baseados em redes permitem representar heterogeneidades individuais, diferentes topologias e outras características que os tornam ainda mais flexíveis que os modelos citados anteriormente [Nowzari et al. 2016, Perez et al. 2019].

Neste contexto, este trabalho propõe o uso de um novo paradigma em que a importância do nó na rede seja obtida com informações provindas da dinâmica da epidemia, e não somente da centralidade da rede. Esta estratégia utiliza as possíveis localizações de onde a epidemia se originou, ou seja, o foco inicial da doença. Dessa forma é possível verificar quais os nós possuem a maior incidência de contágio de acordo com a propagação da doença. Os resultados obtidos mostram que as estratégias propostas se mostram, em média, melhores que as clássicas baseadas em centralidades. Com a inclusão do risco primário em um cenário com dois focos iniciais, mais uma vez as estratégias propostas se mostraram melhores que a estratégia clássica. Uma redução dessa melhora foi observada com o descasamento dos riscos primários entre as fases de alocação e avaliação. $O$ comportamento foi agravado quando os valores associados ao descasamento foi ampliado. Porém, em todos estes cenários, as estratégias propostas se mostraram em média melhores que a baseada em intermediação. O artigo contendo resultados obtidos nesse modelo está em fase de escrita.

\section{Considerações finais}

O potencial das três modelagens abordadas neste trabalho possibilitou o estudo do uso de diversos cenários epidemiológicos, utilizando abordagens determinísticas e estocásticas. Tal potencial pode ser validado pelo estado da arte na atual busca pela mitigação do COVID-19 onde foram utilizados modelos compartimentais [Bastos and Cajueiro 2020], 
MBI [Chang et al. 2020] e redes complexas [Biswas and Sen 2020]. Nesses trabalhos os autores consideraram diferentes métodos de controle, otimização de recursos e inserção de incertezas aos modelos.

As três modelagens diferentes foram utilizadas, neste trabalho, para obtenção de dinâmicas equivalentes entre si, bem como para a busca pelo controle ótimo para mitigação de epidemias. Também possibilitaram a investigação da eficácia de diferentes políticas de controle, no intuito de reduzir a quantidade de indivíduos infectados otimizando o custo envolvido. Ao todo, foram 12 políticas de controle simuladas em 175 cenários. As políticas de controle propostas nas três modelagens utilizadas se mostraram eficientes quanto à redução de indivíduos infectados e obtenção do menor custo possível. Foi possível observar que o uso da avaliação estocástica das políticas de controle proporcionaram analisar cenários mais realistas da estratégia aplicada, se comparado com as obtidas com os modelos clássicos. Adicionalmente, em comparação com modelos compartimentais, o custo computacional para a simulação baseada em indivíduos é tipicamente mais elevada. Assim foi proposto um modelo paralelizado que resultou em uma redução do tempo computacional por um fator de vinte.

Como continuidade deste trabalho propõem-se a utilização dos modelos estudados para definição de políticas de mitigação de outros cenários epidemiológicos. Em especial, foram iniciados estudos de políticas de mitigação para a COVID-19.

\section{Referências}

Anderson, R. M. and May, R. M. (1992). Infectious diseases of humans: Dinamics and control. Oxford University Press.

Bastos, S. B. and Cajueiro, D. O. (2020). Modeling and forecasting the covid-19 pandemic in brazil. arXiv preprint arXiv:2003.14288.

Biswas, K. and Sen, P. (2020). Space-time dependence of corona virus (covid-19) outbreak. arXiv preprint arXiv:2003.03149.

Boccaletti, S., Latora, V., Moreno, Y., Chavez, M., and Hwang, D.-U. (2006). Complex networks: Structure and dynamics. Physics reports, 424(4):175-308.

Casagrandi, R., Bolzoni, L., Levin, S. A., and Andreasen, V. (2006). The sirc model and influenza a. Mathematical Biosciences, 200:156-169.

Chang, S. L., Harding, N., Zachreson, C., Cliff, O. M., and Prokopenko, M. (2020). Modelling transmission and control of the covid-19 pandemic in australia. arXiv preprint arXiv:2003.10218.

Choisy, M., Guégan, and Rohani, P. (2007). Mathematical Modeling of Infectious Diseases Dynamics. ENCYCLOPEDIA OF INFECTIOUS DISEASES: MODERN METHODOLOGIES.

Cisternas, J., Gear, C. W., Levin, S., and Kevrekidis, I. G. (2004). Equation-free modelling of evolving diseases: coarse-grained computations with individual-based models. Proceedings of the Royal Society of London. Series A: Mathematical, Physical and Engineering Sciences, 460(2050):2761-2779. 
Di Giamberardino, P. and Iacoviello, D. (2019). A linear quadratic regulator for nonlinear sirc epidemic model. In 2019 23rd International Conference on System Theory, Control and Computing (ICSTCC), pages 733-738. IEEE.

Emilia, V. and White, R. (2010). An Introduction to Infectious Disease Modelling. Oxford University Press.

Filho, N. A. and Rouquayrol, M. Z. (2006). Introdução à epidemiologia. Guanabara Koogan.

Galvão Filho, A. R., de Lima, T. W., da Silva Soares, A., and Coelho, C. J. (2017). A stochastic approach of sirc model using individual-based epidemiological models. In EPIA Conference on Artificial Intelligence, pages 778-788. Springer.

Galvão Filho, A. R., Galvão, R. K. H., and Yoneyama, T. (2013). Otimização da alocação temporal de recursos para combate a epidemias com transmissão sazonal através de métodos de barreira. Proceeding Series of the Brazilian Society of Computational and Applied Mathematics, 1(1).

Galvão Filho, A. R., Martins de Paula, L. C., Coelho, C. J., de Lima, T. W., and da Silva Soares, A. (2016). Cuda parallel programming for simulation of epidemiological models based on individuals. Mathematical Methods in the Applied Sciences, 39(3):405-411.

Galvăo Filho, A. R., Arruda, F. D. B., Galvăo, R. K. H., and Yoneyama, T. (2011). Programaçăo paralela CUDA para simulaçăo de modelos epidemiológicos baseados em indivíduos. In Em: Anais do X Simpósio Brasileiro de Automaçăo Inteligente, Săo Joăo del-Rei, $M G$.

Kermack, W. and Mckendrick, A. (1927). A contribution to the mathematical theory of epidemics. Proceedings of the Royal Society of London - Series A, 115:700 - 721.

Lambert, S., Gilot-Fromont, E., Toïgo, C., Marchand, P., Petit, É., Garin-Bastuji, B., Gauthier, D., Gaillard, J.-M., Rossi, S., and Thébault, A. (2020). An individual-based model to assess the spatial and individual heterogeneity of brucella melitensis transmission in alpine ibex. Ecological Modelling, 425:109009.

Luenberger, D. G. (2010). Introduction to Dynamic Systems Theory, Models, and Applications. Oxford University Press.

Molter, A., Piovesan, L., Pergher, R., and Varriale, M. (2016). Controle Ótimo em epidemias de dengue. Tema, 17(2).

Nowzari, C., Preciado, V. M., and Pappas, G. J. (2016). Analysis and control of epidemics: A survey of spreading processes on complex networks. IEEE Control Systems, 36(1):26-46.

Perez, I. A., Trunfio, P. A., La Rocca, C. E., and Braunstein, L. A. (2019). Controlling distant contacts to reduce disease spreading on disordered complex networks. Physica A: Statistical Mechanics and its Applications, page 123709.

Siettos, C. I. and Russo, L. (2013). Mathematical modeling of infectious disease dynamics. Mathematical modeling of infectious disease dynamics, 4(4).

Yi-Chia, W., Ching-Sunga, C., and Yu-Jiuna, C. (2020). The outbreak of covid-19 an overview. Journal of the Chinese Medical Association, 83(3). 